\title{
Missed traumatic diaphragmatic hernia and literature review
}

\author{
M Rambau, MB ChB; M M Z U Bhuiyan, DTH, FRCS (Glasgow), MMed \\ Department of General Surgery, Mankweng Hospital, University of Limpopo, Polokwane, South Africa
}

Corresponding author: $M M Z U$ Bhuiyan (bhuiyanmirza@gmail.com)

\begin{abstract}
The diagnosis of traumatic diaphragmatic rupture owing to blunt or penetrating injuries may be missed in acute case presentations. Mortality in patients with acute blunt injuries is highest in those with associated organ damage. The diagnosis of diaphragmatic injuries may be missed because of delayed case presentation or delayed diagnosis. Diaphragmatic injuries should be suspected in all patients with blunt or penetrating injuries of the chest and abdomen. Special care must be taken during the insertion of intercostal drains to the chest in order to avoid iatrogenic injury to the stomach, bowel and other organs, particularly in cases when a missed diaphragmatic injury cannot be excluded. We share in this article our experiences with two cases of missed traumatic diaphragmatic injuries, one on the left and the other on the right side.
\end{abstract}

S Afr Med J 2021;111(11b):1152-1154. https://doi.org/10.7196/SAMJ.2021.v111i11b.16107

The diagnosis of traumatic diaphragmatic rupture owing to blunt or penetrating injuries may be missed in acute case presentation. Surgical treatment is almost always required in order to reduce herniated organs and repair defects. ${ }^{[1]}$ Missed diaphragmatic injuries may occur as a result of delayed case presentation or a delayed diagnosis. ${ }^{[1]}$ The rate of initially missed diaphragmatic injury ranges from $12 \%$ to $66 \%$, and they may even be overlooked during laparotomy. ${ }^{[2]}$ Diaphragmatic injuries should be suspected in all patients with blunt or penetrating traumatic injury of the chest and abdomen. ${ }^{[3]}$ Such injuries were first described in a letter written by Sennertus to Hildan in 1541, who documented the autopsy finding of a herniated stomach through a diaphragmatic injury caused by a self-inflicted stab wound. ${ }^{[4]}$ Diaphragmatic injuries occur more commonly with penetrating trauma than blunt trauma as penetrating trauma is more frequently related to stabbings. ${ }^{[1]}$ Penetrating injuries of the diaphragm tend to be smaller, often measuring less than 2 $\mathrm{cm}$, they are more likely to be occult and often result in a delayed diagnosis. ${ }^{[5]}$ A high index of suspicion must be maintained in order to prevent diagnostic errors of diaphragmatic injuries or related complications, particularly penetrating trauma of the lower chest or upper abdomen. ${ }^{[5,6]} \mathrm{A}$ missed diaphragmatic injury may result in delayed herniation with an increased mortality rate of between $36 \%$ and $48 \%$ when diagnosed late. ${ }^{[6]}$ Here we share our experience of two cases of missed traumatic diaphragmatic injury, one on the left and the other on the right side. Ethical approval was obtained from Pietersburg/Mankweng Research Ethics Committee (ref. no. PMREC 21 JULY UL 2021/B).

\section{Cases}

Case 1

An 18-year-old male patient was referred from a local hospital with a 2-day history of a stab wound in the left chest just below the nipple and an intercostal drain (ICD), which was inserted under the premise of a simple pneumothorax. The patient was transferred to our institution, Mankweng Hospital, because no improvement in condition was observed. Upon arrival at our hospital, the patient was haemodynamically stable. There was a chest laceration in the left 5 th intercostal space with ICD in situ, which had drained $700 \mathrm{~mL}$ of blood. Auscultation revealed decreased air entry on the left side of the chest. The abdomen was not distended, and neither tender nor rigid. The chest X-ray (CXR) (Fig. 1) displayed a left-sided haemothorax and elevated left hemidiaphragm. We diagnosed a diaphragmatic hernia. Subsequent exploratory laparotomy revealed stomach and transverse colon herniated into the chest cavity with an additional grade 1 splenic injury. The diaphragmatic hernia was repaired, ICD repositioned, and the splenic injury did not require surgical intervention. The patient suffered no further complications and was later discharged in good general condition.

\section{Case 2}

A 30-year-old male patient was transferred from a peripheral hospital to Mankweng Hospital, 48 hours after being assaulted, with multiple stab wounds in the chest and abdomen. On examination, the patient was haemodynamically stable, with normal palpable pulses, and no obvious active bleeding observed. Auscultation revealed decreased air entry bilaterally. An ICD had been inserted on the right side

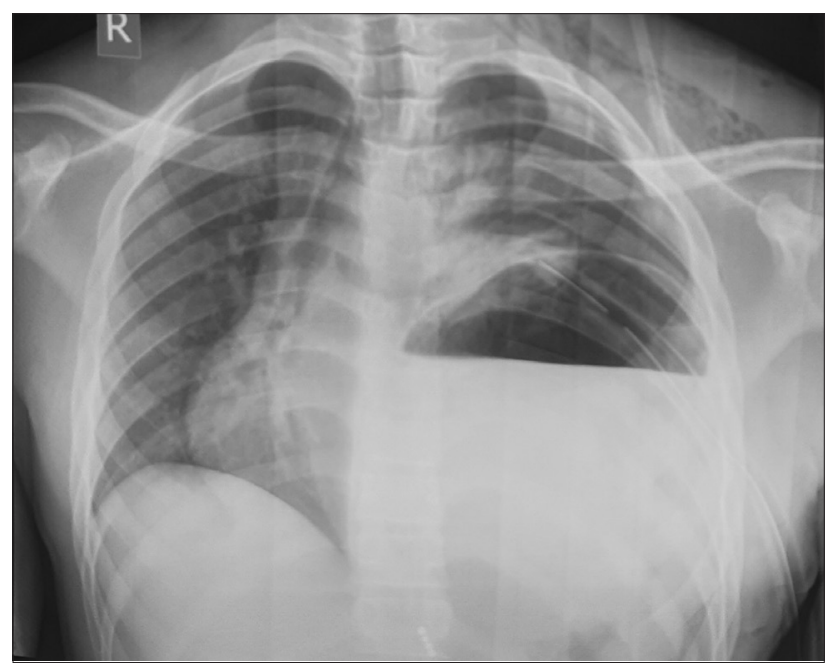

Fig. 1. Chest X-ray displaying a left-sided haemothorax and elevated left hemidiaphragm. 
of the chest. There was a stab wound below the angle of the right scapula, and another stab wound in the left hypochondrium, which had been sutured. The abdomen was peritonitic. The CXR showed a left-sided haemopneumothorax; therefore a second ICD was inserted on the left side. The patient gave consent for exploratory laparotomy. Intraoperatively we found a $2 \mathrm{~cm}$-long perforation of the transverse colon with leaking faeces. The perforation was repaired in the standard way. Post-operatively, on the ward, the patient developed respiratory distress. On the basis of the subsequent CXR (Fig. 2), a right-side diaphragmatic hernia (bowel) was suspected. A computed tomography (CT) scan of the chest (Fig. 3) revealed a right posterior traumatic

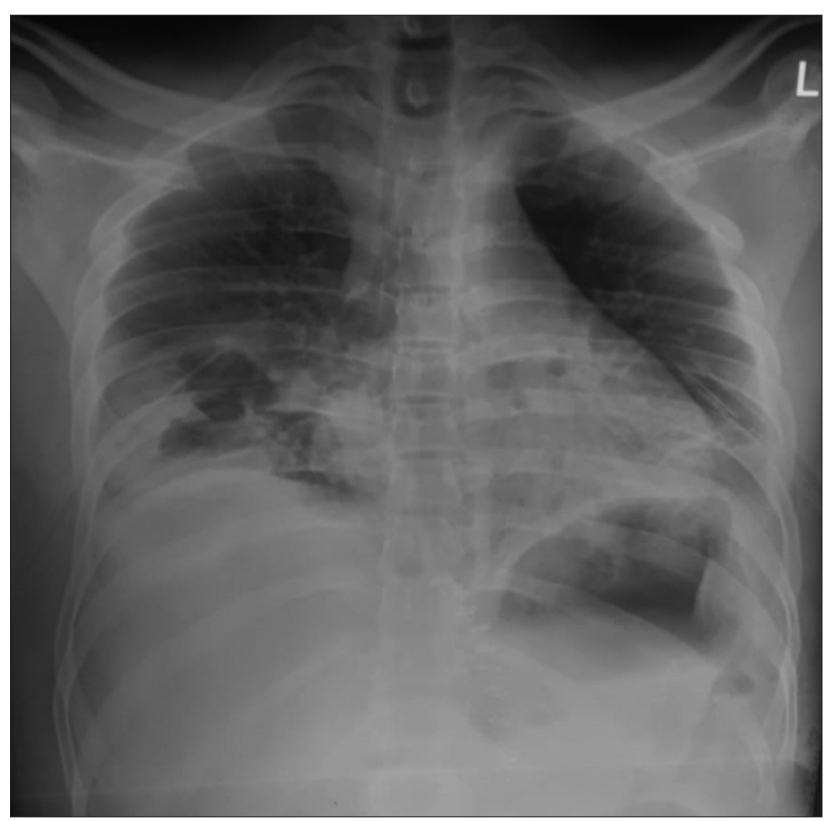

Fig. 2. Chest X-ray suggesting a right-side diaphragmatic hernia of the bowel.

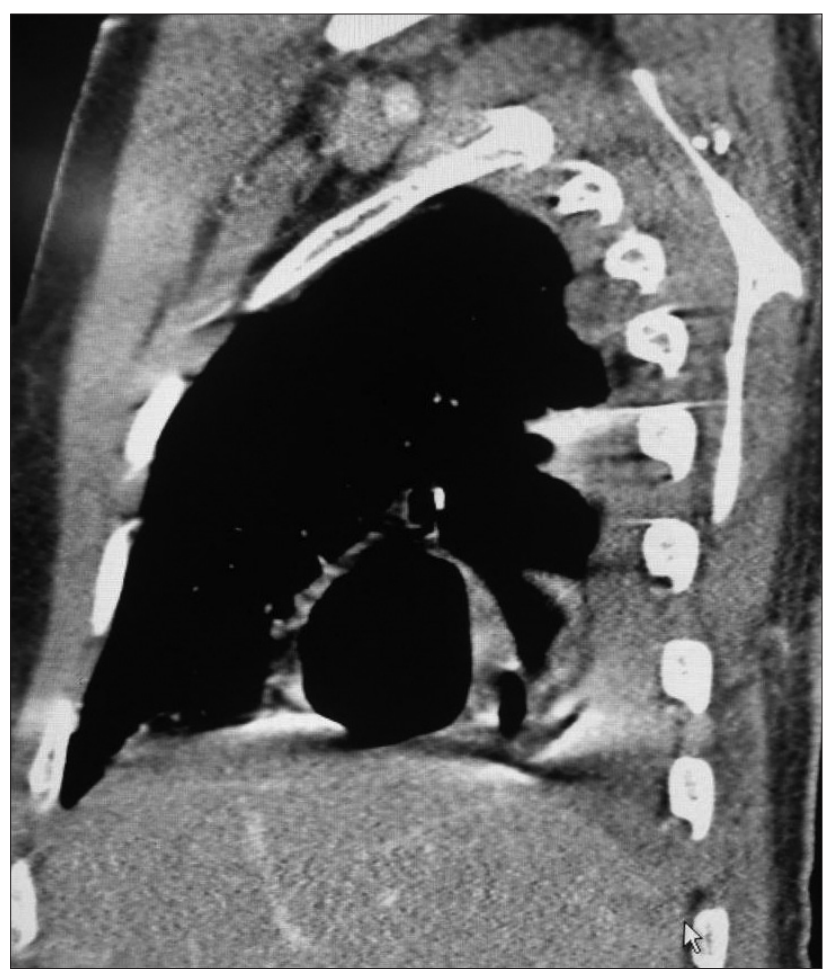

Fig. 3. Computed tomography scan of the chest revealing a right posterior traumatic diaphragmatic hernia. diaphragmatic hernia, bilateral haemopneumothorax with passive atelectasis, a right loculated pneumothorax and grade 2 liver injury. The patient was referred to the cardiothoracic unit of a provincial academic hospital for further management.

\section{Discussion}

The diaphragm is rarely injured alone, and most patients have concomitant abdominal, head or thoracic injuries. Splenic ruptures or liver lacerations are not uncommon injuries in patients with diaphragmatic trauma. ${ }^{[1]}$ Any penetrating injuries to the abdomen or chest from the T4 to T12 dermatome anteriorly and L3 region posteriorly should be considered to have potential diaphragmatic injuries. Left-sided injuries are more common, but small penetrating injuries are more likely to be occult, which frequently result in a delayed diagnosis. ${ }^{[3]}$ Although chest tube insertion is a commonly performed procedure in hospitals and is a lifesaving procedure for a pneumothorax, it may cause perforation of a herniated stomach through a diaphragmatic injury if the diagnosis of diaphragmatic injury is missed. ${ }^{[7]}$ In our first case, simple pneumothorax was incorrectly diagnosed, and an ICD was placed as the definitive treatment. Subsequent lack of clinical improvement resulted in patient transfer to Mankweng Hospital. At our institution, the chest X-ray strongly suggested the presence of diaphragmatic hernia with pneumothorax. Laparotomy revealed that the stomach and transverse colon had herniated into the chest cavity. After reduction of the herniated organs, the appropriate hernia repair was successfully done. The patient recovered without further complications and was discharged home shortly afterwards. Late occult hernias cause more complications requiring more difficult operations, thoracotomy or laparotomy. Blunttrauma-related diaphragmatic ruptures occur when intra-abdominal pressure suddenly rises above the tensile strength of the diaphragmatic tissue. Blunt traumas produce larger, radial tears, often measuring $5-15 \mathrm{~cm} \cdot{ }^{[4]}$ The incidence of right-sided diaphragmatic injuries is low because of the cushioning effects of the liver. When right-sided injuries to the diaphragm are present, the morbidity and mortality rates are higher, owing to other associated injuries being more severe. ${ }^{[8]}$

A history of thoraco-abdominal injuries must be thoroughly obtained from patients, while being aware of possible diaphragmatic injuries. A high index of suspicion and use of radiological investigations is essential for prompt correct diagnosis. ${ }^{[6]}$ Delayed diagnosis is common, and it can occur as late as 15 years following an initial injury. ${ }^{[9]}$ If the correct diagnosis is missed, patients may be referred later with herniation of bowel contents into the chest, obstructions, or strangulations of intra-abdominal organs. ${ }^{[1]}$ Herniation on the right side is missed more commonly, ${ }^{[6,10]}$ as was evident in our second case.

Intra-abdominal injuries may be excluded by a detailed clinical examination along with radiological investigations, and if available, a minimally invasive surgical approach. The placement of a nasogastric tube may also be helpful in making the diagnosis. Injuries to the diaphragm do not heal spontaneously; therefore operative repair is always required for left-sided injuries, and for most right-sided injuries. ${ }^{[8]}$

A review of delayed presentation of diaphragmatic ruptures found chest radiographs for left-sided injuries were diagnostic in $46 \%$ of patients, and in $17 \%$ for right-sided.$^{[8]}$ On occasion, the CXR might show the pathognomic signs of a diaphragmatic injury, such as a nasogastric tube within the chest, hepatic displacement into the right hemithorax or herniated bowel loops inside the chest, with or without focal constrictions of the viscus at the herniation site. Herniations at the costophrenic angle may be misdiagnosed on chest radiograph, appearing as pleural effusions or a haemothorax. CT scans are very useful in detecting diaphragmatic injuries. ${ }^{[6]}$ 
With increasing utilisation of laparoscopy and thoracoscopy, more diaphragmatic injuries are diagnosed correctly. In a 2007 study, ${ }^{[6]}$ the diagnostic accuracy of thoracoscopy in occult diaphragmatic injuries after penetrating injury was $100 \%$. Laparotomy appears to be more appropriate in cases with haemodynamic instability, peritonitis and when intra-abdominal lesions are suspected. During laparotomy, regardless of the suspected diagnosis, full examination of the whole diaphragm should be undertaken. ${ }^{[4]}$ The use of non-absorbable sutures is widely recommended. Interrupted and continuous techniques are equally effective and, while simple sutures are sufficient in small defects, large defects require a synthetic mesh. ${ }^{[6,11]}$

\section{Conclusion}

A high index of suspicion must be maintained in order to prevent the incorrect diagnosis and complications of missed diaphragmatic injuries. These injuries may result in herniation or strangulation of abdominal organs into the thoracic cavity through traumatic defects in the diaphragm. Proper care needs to be taken when inserting ICDs in order to avoid iatrogenic injury of the stomach, bowel and other organs, especially in cases of suspected diaphragmatic injuries.

\section{Declaration. None.}

Acknowledgements. None.

Author contributions. Both authors made substantial contributions to the following: data gathering and literature research; and gaining ethical approval. MR was responsible for initial writing of the manuscript and MMZUB for final writing and editing.

\section{Funding. None.}

Conflicts of interest. None.

1. Corbellini C, Costa S, Canini T, Villa R, Avesani EC. Diaphragmatic rupture: A single-institution experience and literature review. Ulus Travma Acil Cerrahi Derg 2017;23(5):421-426. https://doi.org/10.5505/ tites.2017.78027

2. Yilmaz M, Isik B, Ara C, et al. Gastric perforation during chest tube placement for acute diaphragmatic Hanna WC, Ferri LE, Fata P, Razek T, Mulder DS. The current status of traumatic diaphragmatic injury. Ann . Hanna WC, Ferri LE, Fata P, Razek T, Mulder DS. The current status of traumatic dia
Thorac Surg 2008;8(3):1044-1048. https://doi.org/10.1016/j.athoracsur.2007.10.084

4. Bosanquet D, Farboud A, Luckraz H. A review diaphragmatic injury. Respir Med CME 2009;2(1):1-6. https://doi.org/10.1016/j.rmedc.2009.01.002;

5. Gooseman MR, Rawashdeh M, Mattam K, Rao JN, Vaughan PR, Edwards JG. Unifying for trans diaphragmatic intercostal hernia and other costal margin injuries. Eur J Cardiothoracic Surg 2019;56(1):150158. https://doi.org/10.1093/ejtts/ezzo20

6. Balaguera J, García-Almenta M, Segovia J, et al. Delayed asymptomatic left traumatic diaphragmatic hernia after abdominal stab wounds. Internet J Surg 2007;17(2). https://ispub.com/IIS/17/2/7958 (accessed 5 September 2021)

7. Kar SK, Ganguly T, Nayek J. Emergency chest tube insertion in a case of thoracic trauma with hidden diaphragmatic rupture resulted in injury of herniated stomach: A case report. EC Anaesthesia 2015;2:47-51. 8. Rashid F, Chakrabarty MM, Singh R, Iftikhar SY. A review on delayed presentation of diaphragmatic rupture. World J Emerg Surg 2009;4:32. https://doi.org/10.1186/1749-7922-4-32

9. Symbas PN, Vlasis SE, Hatcher $\mathrm{C}$ Jr. Blunt and penetrating diaphragmatic injuries with or without herniation of organs into the chest. Ann Thorac Surg 1986;42:158-162. https://doi.org/10.1016/s0003-4975(10)60510-3

10. Sirbu H, Busch T, Spillner I, Schachtrupp A, Autschbach R. Late bitateral diaphragmatic rupture: Challenging diagnostic and surgical repair. Hernia 2005;9:90-92. https://doi.org/10.1007/s10029-004-0243-4

11. Matsevych OY. Blunt diaphragmatic rupture: Four years' experience. Hernia 2008;12(1):73-78. https://doi. org/10.1007/s10029-007-0283-7

Accepted 5 September 2021 